OPEN ACCESS

Edited by:

Nuno Sousa,

University of Minho, Portugal

Reviewed by:

Andreia Teixeira-Castro, University of Minho, Portugal Andre Brown Imperial College London, UK

*Correspondence: Christoph Schmitz christoph_schmitz@ med.uni-muenchen.de

Received: 29 June 2015 Accepted: 14 March 2016 Published: 31 March 2016

Citation:

Angstman NB, Frank $\mathrm{H}-\mathrm{G}$ and Schmitz C (2016) Advanced Behavioral Analyses Show that the Presence of Food Causes Subtle Changes in C. elegans Movement. Front. Behav. Neurosci. 10:60. doi: 10.3389/fnbeh.2016.00060

\section{Advanced Behavioral Analyses Show that the Presence of Food Causes Subtle Changes in C. elegans Movement}

\author{
Nicholas B. Angstman, Hans-Georg Frank and Christoph Schmitz * \\ Department of Neuroanatomy, Ludwig-Maximilians University of Munich, Munich, Germany
}

As a widely used and studied model organism, Caenorhabditis elegans worms offer the ability to investigate implications of behavioral change. Although, investigation of C. elegans behavioral traits has been shown, analysis is often narrowed down to measurements based off a single point, and thus cannot pick up on subtle behavioral and morphological changes. In the present study videos were captured of four different C. elegans strains grown in liquid cultures and transferred to NGM-agar plates with an E. coli lawn or with no lawn. Using an advanced software, WormLab, the full skeleton and outline of worms were tracked to determine whether the presence of food affects behavioral traits. In all seven investigated parameters, statistically significant differences were found in worm behavior between those moving on NGM-agar plates with an E. coli lawn and NGM-agar plates with no lawn. Furthermore, multiple test groups showed differences in interaction between variables as the parameters that significantly correlated statistically with speed of locomotion varied. In the present study, we demonstrate the validity of a model to analyze $C$. elegans behavior beyond simple speed of locomotion. The need to account for a nested design while performing statistical analyses in similar studies is also demonstrated. With extended analyses, C. elegans behavioral change can be investigated with greater sensitivity, which could have wide utility in fields such as, but not limited to, toxicology, drug discovery, and RNAi screening.

Keywords: C. elegans, E. coli, food, locomotion, tracking

\section{INTRODUCTION}

As a highly-used model organism, Caenorhabditis elegans (C. elegans) worms offer a wide range of possibilities for scientific research. With advantages including, but not limited to, easy maintenance and handling, forward and reverse genetic manipulability (Jorgensen and Mango, 2002), and a fully mapped nervous system connectome of 302 neurons (White et al., 1986), C. elegans offer an alternative to larger rodent model organisms. Furthermore, C. elegans can be cultured in both liquid media and on agar plates (Sulston and Brenner, 1974; Angstman et al., 2015) and fed with concentrated Escherichia coli (E. coli).

Behavioral analysis of C. elegans is generally performed on NGM-agar plates in a quasi 2D model under a dissecting microscope using video capturing software, although assays measuring behavior in liquid have been demonstrated (Hardaway et al., 2014; Restif et al., 2014), as have 
assays in behavioral arenas (Albrecht and Bargmann, 2011). As NGM-agar plate behavior assays remain the status quo, various tracking software is available to generate a readout on such behavior (summarized in Husson et al., 2012). Features of such software range from single worm tracking to multi-worm tracking, and from single point (centroid) tracking to full worm outline tracking (Table 1). Although, readouts using methods are often limited to speed of locomotion, more complicated behavioral phenotypes such as omega bending and reversals have been defined and detected (Huang et al., 2006). Some authors have attempted to identify as many as 702 features of worm movement by splitting certain features into sub-features (for example breaking up tail motion direction into forward, paused, and negative tail motion direction; Yemini et al., 2013).

As C. elegans are often raised on NGM-agar plates with an E. coli lawn, behavioral assays can be performed in the presence of food or on bare NGM-agar plates. The presence of food has previously been shown to decrease C. elegans speed of locomotion (Ramot et al., 2008). Thus, the effect of an E. coli lawn in a behavioral assay is of relevance to, at the very least, investigations of feeding related genes (see, e.g., de Bono and Bargmann, 1998).

In the present study we demonstrate that statistically significant behavioral differences beyond speed of locomotion can be observed when investigating four different strains of C. elegans on either NGM-agar plates with an E. coli lawn or with no lawn. Furthermore, we show that such a change in environment also affects the relationship of speed with other behavioral traits-that is behavioral change can be observed even in the lack of an observed change in speed of locomotion. Specifically we tested the hypothesis that various behavioral traits can be measured to show the effect of the presence of $E$. coli in the moving properties of C. elegans.

\section{MATERIALS AND METHODS}

\section{Nematodes}

Wild type (N2, Bristol), DA508 npr-1(n1353), DA609 $n p r-1$ (ad609), and CX4148 npr-1(ky13) C. elegans, as well as OP50 E. coli were obtained from the Caenorhabditis Genetics
Center (Minneapolis, MN, USA), which is funded by NIH Office of Research Infrastructure Programs (P40 OD010440). Mutant $n$ pr-1 strains of $C$. elegans were selected based on previous findings that such strains are hyperactive on plates containing food as compared to wild type worms (de Bono and Bargmann, 1998). From a stock liquid culture, synchronous young adult worms were produced via sodium hypochlorite treatment and sucrose cleaning as described in detail in Angstman et al. (2015). Worms were raised in liquid cultures at $24^{\circ} \mathrm{C}$ in an incubated shaker (NB 205V, N Biotek, Bucheon, South Korea).

\section{Assay}

From a tube of worms in S-Medium, $10 \mu$ l containing 15-30 worms were pipetted on to a modified membrane filter-vacuum filtration system (Angstman et al., 2015). Following removal of liquid, membrane filters were flipped and placed on to a 6 cm NGM-agar plate (see Supplemental Video 2 in Angstman et al., 2015). Plates contained either an E. coli lawn (created by spreading over the agar surface and allowing to grow overnight) or no lawn (control).

Plates were immediately placed under a dissecting microscope (MZ75; Leica, Wetzlar, Germany; equipped with 1.0X PlanApo objective) with an LCD light source with a color temperature of $2800 \mathrm{~K}$ (KL 1500; Schott, Mainz, Germany). Using the video capture function of the software, WormLab (Version 3.0.0, MBF Bioscience, Williston, VT, USA), $60 \mathrm{~s}$ long videos with a resolution of $1280 \times 960$ pixels were taken at 15 frames/s using a mono digital camera (Grasshopper 2, Point Grey Research, Richmond, BC, Canada). Using a horizontal $\mathrm{mm}$ ruler and the measure function of WormLab, videos were determined to have a scale of $8.37 \mu \mathrm{m} /$ pixel, which enabled precise and accurate investigation of the parameters described in the next section. Accordingly, the field-of-view of the camera was 10.7 by $8.0 \mathrm{~mm}$ and, thus, 3\% of the base area of the agar plates (Figure 1 and Supplementary Videos S1, S2).

At the beginning of each video plates were adjusted so that all worms were within the field-of-view of the camera. All worms that were tracked for $>75$ of the first 150 frames were included in the final analysis, with track lengths of up to 900 frames possible. Tracks that started after the first 150 frames were excluded from

TABLE 1 | Summary of $C$. elegans tracking software features.

\begin{tabular}{|c|c|c|c|c|c|c|c|c|c|}
\hline Tracker & Nemo $^{a}$ & $\begin{array}{l}\text { The parallel } \\
\text { worm Tracker }\end{array}$ & OptoTracker ${ }^{\mathrm{C}}$ & $\begin{array}{l}\text { Multimodal } \\
\text { illumination and } \\
\text { tracking system }\end{array}$ & CoLBeRT $^{e}$ & $\begin{array}{l}\text { The multi } \\
\text { worm tracker }\end{array}$ & $\begin{array}{l}\text { Optomechanical } \\
\text { system for } \\
\text { virtual } \\
\text { environments }\end{array}$ & $\begin{array}{l}\text { Worm Tracker } \\
2.0^{\mathrm{h}}\end{array}$ & WormLab ${ }^{i}$ \\
\hline $\begin{array}{l}\text { Single/ } \\
\text { Multiple } \\
\text { worms }\end{array}$ & Single & Multiple & Multiple & Single & Single & Multiple & Single & Single & Multiple \\
\hline $\begin{array}{l}\text { Tracking } \\
\text { capability }\end{array}$ & $\begin{array}{l}\text { Skeleton } \\
\text { and } \\
\text { outline }\end{array}$ & Mid-point & Mid-point & Skeleton and outline & $\begin{array}{l}\text { Skeleton and } \\
\text { outline }\end{array}$ & $\begin{array}{l}\text { Skeleton and } \\
\text { outline }\end{array}$ & Bright spot & $\begin{array}{l}\text { Skeleton and } \\
\text { outline }\end{array}$ & $\begin{array}{l}\text { Skeleton and } \\
\text { outline }\end{array}$ \\
\hline
\end{tabular}

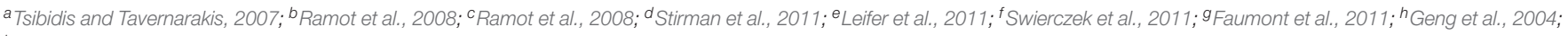
${ }^{i}$ Roussel et al., 2014. 


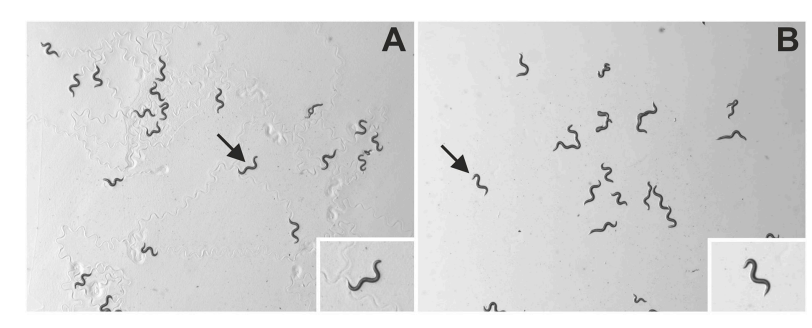

FIGURE 1 | Representative images of C. elegans as analyzed in the present study. The panels show unprocessed frames of videos of wild type (N2) C. elegans on NGM-agar plates with an E. coli lawn (A) or with no lawn (B). The insets show individual worms (indicated by arrows) at higher magnification. The slightly uneven illumination of the panels stems from the fact that in the experimental setup of the present study, the field-of-view was placed offset from the middle of the agar plates. However, this had no impact on unequivocal identification and tracking of the worms on the plates (evaluation data not shown).

the analysis because they could have represented worms that initially moved out of but then came back into the field-of-view of the camera. These parameters avoided the possibility of double counting individual worms.

Captured videos were tracked with WormLab using settings outlined in Supplementary Table 1. Results from each video including Position-Midpoint (x,y), Bending Angle-Mid-Point, Wavelength, Omega Bend, and Reversal were exported and processed using Microsoft Excel 2010 (Microsoft, Redmond, WA, USA).

\section{Investigated Parameters Speed of Locomotion}

Speed of worms was calculated from Position to Midpoint ( $\mathrm{x}, \mathrm{y})$ data as in our earlier study (Angstman et al., 2015). Direction was not taken into account. Using coordinates, speed was calculated for each second as the distance traveled over 15 frames. Single point values of $>500 \mu \mathrm{m} / \mathrm{s}$ were considered outliers and removed from the data set. Each qualifying worm was represented with one value for average speed determined by the arithmetic mean of the values calculated each second.

\section{Bending Angle}

The bending angle is defined as the angle between the midpointhead and midpoint-tail segments, with a straight worm set as zero degrees (Figure 2A). For a given worm, a bending angle was measured for each frame tracked. In the final readout of average bending angle for a given worm, the absolute value of the angle measured in each frame was averaged. Standard deviation of all absolute value bending angles was calculated for each qualifying worm.

\section{Omega Bending}

As defined by WormLab, omega bending occurs when the bending angle exceeds 90 degrees and continues until the bending angle goes below $90^{\circ}$ (Figure 2B). Omega bending was counted only when a minimum duration of 10 frames was reached. For

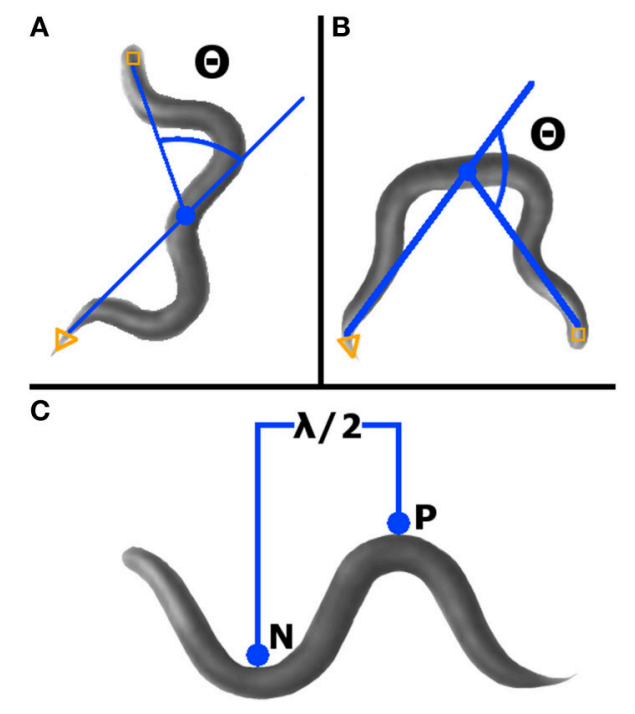

FIGURE 2 | WormLab advanced analyses. (A) Bending angle $(\Theta)$ was measured as the supplement of the angle between the head, mid-point, and tail. Worms with head, mid-point, and tail in line have an angle of zero. (B) Omega bending is indicated when the angle $(\Theta)$ created between the head, mid-point, and tail is $<90^{\circ}$ (i.e., the bending angle is $>90^{\circ}$ ). (C) Wavelength was measured as two times the distance between the positive $(P)$ and negative $(\mathrm{N})$ stationary points.

each worm meeting qualifying requirements, omega bending was expressed as a percentage of frames tracked.

\section{Wavelength}

This parameter is defined as the two times the measurement between the positive and the negative inflection points (Figure 2C). Wavelength was calculated for each worm in each frame tracked, except when at zero or only one inflection point was found. For qualifying worms, average wavelength was expressed as the average of all calculated wavelengths (i.e., from each frame tracked).

\section{Reversal Frequency}

This parameter was calculated based on smoothed speed provided by WormLab, a smoothed moving average speed taken over a 20 frame span (based on Cleveland and Devlin, 1988). In order to remove bias from possible incorrect head/tail recognition, smoothed speed was inversed if over half of the frames tracked were measured as negative speed values. Reversal was then counted only in stretches of negative smoothed speed $\geq 10$ frames. Final results were reported for each qualifying worm as a percentage of reversals per frame tracked.

\section{Statistical Analysis}

In the final analyses of NGM-agar plates with an E. coli lawn, 172 N2 worms on 10 different plates, $189 n$ pr-1(n1353) worms on six different plates, $132 n p r-1$ (ad609) worms on six different plates and 129 npr-1(ky13) worms on six different plates were counted. Analyses of NGM-agar plates with no lawn included 173 $\mathrm{N} 2$ worms on 10 different plates, $159 n p r-1(n 1353)$ worms on six 
different plates, 139 npr-1(ad609) worms on six different plates, and $167 n p r-1(k y 13)$ worms on six different plates.

Due to the fact that several worms were analyzed per plate, a nested ANOVA design was used to determine the significance of (i) lawn type (i.e., plates with an E. coli lawn vs. plates with no lawn) and (ii) whether the nested nature of this assay contributed to observed differences (c.f. Aarts et al., 2014). In the nested ANOVA, the various investigated parameters were used as the dependent variable, while lawn type was a fixed factor, and plate number was a random factor.

To investigate correlation between analyses, the runs test (c.f. Wald and Wolfowitz, 1940) was used to determine if relationships departed from linearity. If a statistically significant departure was not found, linear regression was used to compare analyses. If departure from linearity was statistically significant, non-parametric correlation (Spearman) was used.

Nested ANOVA was performed in SPSS (Version 23 for Windows; IBM, Armonk, NY, USA), while all other statistical analyses were performed in GraphPad Prism (version 5.04 for Windows; GraphPad Software, San Diego, CA, USA).

A $p$-value of 0.05 was used as the criterion for statistical significance in all analyses.

\section{RESULTS}

\section{Lawn vs. No Lawn}

The results of comparisons between worms on NGM-agar plates with an E. coli and worms on NGM-agar plates with no lawn are summarized in detail in Table 2. Lawn type was found to result

TABLE 2 | Summary of analyses and comparison between C. elegans on plates with E. coli and no lawn.

\begin{tabular}{|c|c|c|c|c|c|c|c|c|c|c|c|c|c|}
\hline \multirow[t]{2}{*}{ Analysis } & \multirow[t]{2}{*}{ Strain } & \multicolumn{4}{|c|}{ Lawn Type } & & & & & & & & \\
\hline & & Mean & $S D$ & Mean & $S D$ & df1 & $d f 2$ & $\boldsymbol{F}$ & $p$ & $d f 1$ & $d f 2$ & $\boldsymbol{F}$ & $p$ \\
\hline \multirow[t]{2}{*}{ Average speed $(\mu \mathrm{m} / \mathrm{s})$} & N2 & 128.6 & 41.6 & 146.9 & 55.3 & 1 & 19.324 & 4.4 & 0.049 & 18 & 325 & 2.394 & 0.001 \\
\hline & $n p r-1(n 1353)$ & 110.8 & 39.6 & 130.4 & 49.0 & 1 & 10.226 & 5.2 & 0.045 & 10 & 336 & 3.679 & 0.000 \\
\hline \multirow[t]{4}{*}{ Average Angle $\left(^{\circ}\right)$} & N2 & 34.3 & 14.2 & 40.0 & 17.1 & 1 & 19.387 & 6.3 & 0.022 & 18 & 325 & 2.287 & 0.002 \\
\hline & $n p r-1(n 1353)$ & 25.0 & 12.7 & 44.8 & 20.5 & 1 & 10.248 & 38.0 & 0.000 & 10 & 336 & 3.359 & 0.000 \\
\hline & $n p r-1$ (ad609) & 28.2 & 12.1 & 48.9 & 24.3 & 1 & 11.108 & 98.6 & 0.000 & 10 & 259 & 0.776 & 0.652 \\
\hline & $n p r-1(k y 13)$ & 26.7 & 11.1 & 36.1 & 17.7 & 1 & 12.138 & 42.5 & 0.000 & 10 & 298 & 0.568 & 0.840 \\
\hline & $n p r-1(k y 13)$ & 18.1 & 6.6 & 25.6 & 12.7 & 1 & 11.453 & 36.9 & 0.000 & 10 & 298 & 0.824 & 0.606 \\
\hline \multirow[t]{4}{*}{ Average wavelength $(\mu \mathrm{m})$} & N2 & 383.0 & 42.7 & 347.5 & 43.9 & 1 & 19.931 & 36.9 & 0.000 & 18 & 325 & 1.653 & 0.046 \\
\hline & $n p r-1(n 1353)$ & 363.2 & 52.0 & 330.0 & 53.3 & 1 & 10.398 & 15.7 & 0.002 & 10 & 336 & 2.097 & 0.024 \\
\hline & $n p r-1$ (ad609) & 346.1 & 70.2 & 318.2 & 67.3 & 1 & 10.580 & 6.3 & 0.030 & 10 & 259 & 1.465 & 0.153 \\
\hline & $n p r-1(k y 13)$ & 411.3 & 45.2 & 371.8 & 60.9 & 1 & 10.819 & 29.0 & 0.000 & 10 & 298 & 1.441 & 0.161 \\
\hline \multirow[t]{4}{*}{ St. Dev. wavelength $(\mu \mathrm{m})$} & N2 & 103.3 & 31.3 & 91.8 & 34.3 & 1 & 19.533 & 4.6 & 0.045 & 18 & 325 & 2.073 & 0.007 \\
\hline & $n p r-1(n 1353)$ & 103.4 & 33.4 & 90.9 & 30.8 & 1 & 10.419 & 6.8 & 0.025 & 10 & 336 & 1.996 & 0.033 \\
\hline & npr-1 (ad609) & 85.0 & 31.6 & 99.3 & 37.8 & 1 & 10.451 & 6.6 & 0.027 & 10 & 259 & 1.880 & 0.048 \\
\hline & $n p r-1(k y 13)$ & 93.1 & 31.9 & 86.1 & 35.4 & 1 & 10.803 & 3.1 & 0.108 & 10 & 298 & 1.468 & 0.150 \\
\hline & npr-1(ad609) & 8.22 & 12.22 & 21.63 & 13.23 & 1 & 10.566 & 49.7 & 0.000 & 10 & 259 & 1.501 & 0.139 \\
\hline & $n p r-1(k y 13)$ & 2.24 & 6.77 & 4.68 & 10.41 & 1 & 11.830 & 8.4 & 0.014 & 10 & 298 & 0.659 & 0.762 \\
\hline
\end{tabular}

$P$-values smaller than 0.05 are given boldface. 
in a statistically significant difference in 25/28 analyses across the four strains of C. elegans.

In all experiments carried out, worms showed substantial interindividual variation in all investigated parameters. For example, speed of locomotion of $\mathrm{N} 2$ worms on plates with an E. coli lawn varied between 5.5 and $221 \mu \mathrm{m} / \mathrm{s}$, and on plates with no lawn between 4.0 and $255 \mu \mathrm{m} / \mathrm{s}$ (Figure 3). Besides this, mean values of all investigated parameters showed considerable inter-plate variability. For example, mean speed of locomotion of $\mathrm{N} 2$ worms on plates with an E. coli lawn varied between 106 and $155 \mu \mathrm{m} / \mathrm{s}$ among plates, and on plates with no lawn between 112 and $186 \mu \mathrm{m} / \mathrm{s}$ among plates (Figure 3).

\section{Plate Effect}

The nested factor-accounting for the fact that multiple specimen can be grouped by plate-accounted for statistically significant difference in 13/28 analyses (all results of statistical analysis are summarized in Table 2). Both lawn type and the nested factor were statistically significant in 12 cases. Lawn type, but not the nested factor, was statistically significant in 13 cases, while the nested factor, but not lawn type, was statistically significant in only one case. In only two cases was neither lawn type nor the nested factor statistically significant.

\section{Correlation of Speed with Various Behavioral Analyses}

The results of these analyses are summarized in Table 3 for $C$. elegans on plates with an E. coli lawn and in Table 4 for C. elegans on plates with no lawn. When comparing average speed to other analyses in worms on an E. coli lawn, statistically significant departure from linearity was found in only five cases out of 24 (six correlations across four strains). Departure from linearity was observed in $8 / 24$ cases for worms on plates with no lawn. Linear regression and Spearman non-linear correlation yielded statistical significance in 12 cases for worms on NGM-agar plates with an E. coli lawn and 20 cases for worms on NGM-agar plates with no lawn.

\section{DISCUSSION}

\section{Summary of Results}

The main results of the present study can be summarized as follows: advanced C. elegans behavioral traits, in both wild type and mutant strains, can be used to demonstrate behavioral differences between test groups. Furthermore, such behavioral traits go beyond the standard measure of speed of locomotion and often either do not significantly correlate statistically with speed of locomotion or only correlate with speed of locomotion within certain test groups. Only one analysis, reversal percentage, was found to correlate with average speed of locomotion in all cases. High interindividual variability within analyses was observed, in line with our previously shown data (Angstman et al., 2015), and inter-plate variability was also shown to be a factor in some differences seen in the analyses. This reinforces the need for large sample sizes for behavioral analyses of C. elegans, and also shows the importance of using nested analyses when assaying behavior of multiple worms per NGM-agar plate. Using the methods demonstrated in the present study, the ability to accurately measure advanced traits of $C$. elegans behavior offers enhanced functionality in C. elegans research.

\section{Relevance}

The comparison of C. elegans worms moving on NGM-agar plates with an E. coli lawn to worms moving on NGM-agar plates with no lawn represents a relevant biological example for behavioral change in C. elegans. This methodology has been used previously using behavioral analysis on N2 worms (Schwarz et al., 2015). The $n p r-1$ strains used in the present study are described in this context as well. This methodology and these strains were, for example, used to identify genes that play a role in feeding in $C$. elegans (de Bono and Bargmann, 1998; de Bono et al., 2002). The behavioral traits used in the present study were also identified as characteristics of $C$. elegans behavior in the literature (e.g., Buckingham and Sattelle, 2008).

At first glance, it seems that the results of the present study are not in line with certain data reported by de Bono and Bargmann (1998) and de Bono et al. (2002). For example, de Bono and Bargmann (1998) found an increase in mean speed of locomotion

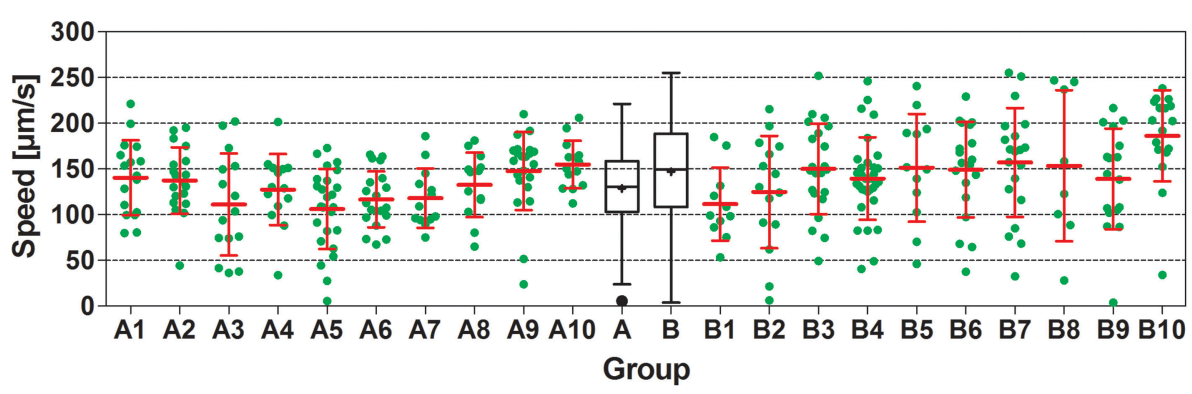

FIGURE 3 | Speed of locomotion of N2 (wild type) C. elegans on NGM agar plates with an E. coli lawn and on plates with no lawn. Groups A1-A10 and B1-B10 show individual data (green dots) and mean \pm standard deviation (red lines) of speed of locomotion of N2 worms on 10 plates with an E. coli lawn (Groups A1-A10) and on 10 plates with no lawn (Groups B1-B10). Groups A and B show Tukey boxplots of the speed of locomotion of all worms on plates with an E. coli lawn (Group A) and of all worms on plates with no lawn (Group B). Nested ANOVA showed a statistically significant difference between worms on plates with an E. coli lawn and worms on plates with no lawn $(p=0.049)$ as well as a statistically significant effect among plates $(p=0.001$; see also Table 2). 
TABLE 3 | Summary of correlation between analyses for worms on plates with an E. coli lawn.

\begin{tabular}{|c|c|c|c|c|c|c|c|c|c|}
\hline \multirow[t]{2}{*}{ Calculation } & \multirow[t]{2}{*}{ Strain } & \multicolumn{3}{|c|}{ Runs test } & \multicolumn{3}{|c|}{ Linear regression } & \multicolumn{2}{|c|}{ Non-linear correlation } \\
\hline & & Points Above line & Points Below line & $p$ & $r^{2}$ & $\boldsymbol{F}$ & $p$ & Spearman & $p$ \\
\hline \multirow{4}{*}{$\begin{array}{l}\text { Average speed vs. } \\
\text { average angle }\end{array}$} & N2 & 72 & 100 & 0.917 & 0.030 & 5.277 & 0.023 & & \\
\hline & npr-1(n1353) & 74 & 115 & 0.242 & 0.093 & 19.11 & $<0.001$ & & \\
\hline & npr-1(ad609) & 55 & 77 & 0.315 & 0.01949 & 2.585 & 0.110 & & \\
\hline & npr-1(ky13) & 56 & 73 & 0.508 & 0.232 & 38.43 & $<0.001$ & & \\
\hline \multirow{4}{*}{$\begin{array}{l}\text { Average speed vs. St. } \\
\text { Dev. angle }\end{array}$} & N2 & 76 & 96 & 0.810 & 0.001 & 0.232 & 0.631 & & \\
\hline & $n p r-1(n 1353)$ & 74 & 115 & 0.699 & 0.014 & 2.697 & 0.102 & & \\
\hline & npr-1(ad609) & 52 & 80 & 0.792 & 0.002 & 0.310 & 0.579 & & \\
\hline & $n p r-1(k y 13)$ & 62 & 67 & 0.766 & 0.014 & 1.824 & 0.179 & & \\
\hline \multirow{4}{*}{$\begin{array}{l}\text { Average speed vs. } \\
\text { average wavelength }\end{array}$} & N2 & 83 & 89 & 0.129 & 0.006 & 1.042 & 0.309 & & \\
\hline & $n p r-1(n 1353)$ & 98 & 91 & 0.962 & 0.108 & 22.61 & $<0.001$ & & \\
\hline & npr-1(ad609) & 67 & 65 & 0.397 & 0.403 & 87.78 & $<0.001$ & & \\
\hline & npr-1(ky13) & 67 & 62 & 0.577 & 0.207 & 33.06 & $<0.001$ & & \\
\hline \multirow{4}{*}{$\begin{array}{l}\text { Average speed vs. St. } \\
\text { Dev. wavelength }\end{array}$} & N2 & 87 & 85 & 0.649 & 0.178 & 36.93 & $<0.001$ & & \\
\hline & $n p r-1(n 1353)$ & 87 & 102 & 0.214 & 0.108 & 22.73 & $<0.001$ & & \\
\hline & npr-1(ad609) & 60 & 72 & 0.301 & 0.017 & 2.219 & 0.139 & & \\
\hline & npr-1(ky13) & 64 & 65 & 0.430 & 0.016 & 2.048 & 0.155 & & \\
\hline \multirow{4}{*}{$\begin{array}{l}\text { Average speed vs. omega } \\
\text { bending Pct }\end{array}$} & $\mathrm{N} 2$ & 25 & 147 & 0.258 & 0.006 & 0.989 & 0.322 & & \\
\hline & $n p r-1(n 1353)$ & 45 & 144 & $<0.001$ & & & & -0.098 & 0.178 \\
\hline & npr-1(ad609) & 17 & 115 & 0.344 & 0.008 & 1.084 & 0.300 & & \\
\hline & npr-1(ky13) & 22 & 107 & $<0.001$ & & & & 0.127 & 0.152 \\
\hline \multirow{4}{*}{$\begin{array}{l}\text { Average speed vs. } \\
\text { reversal Pct }\end{array}$} & N2 & 54 & 118 & 0.005 & & & & -0.448 & $<0.001$ \\
\hline & npr-1(n1353) & 60 & 129 & $<0.001$ & & & & -0.363 & $<0.001$ \\
\hline & npr-1(ad609) & 45 & 87 & 0.064 & 0.202 & 32.82 & $<0.001$ & & \\
\hline & npr-1(ky13) & 32 & 97 & $<0.001$ & & & & -0.314 & 0.003 \\
\hline
\end{tabular}

$P$-values smaller than 0.05 are given boldface.

of wildtype (N2) C. elegans of $\sim 110 \mu \mathrm{m} / \mathrm{s}$ on plates with an E. coli lawn to $\sim 300 \mu \mathrm{m} / \mathrm{s}$ on plates with no lawn $(+170 \%$; in de Bono et al.'s (2002) study this difference was $+225 \%)$. This difference is much greater than what was found in the present study (the mean speed of locomotion of N2 C. elegans was 128.6 $\mu \mathrm{m} / \mathrm{s}$ on plates with an E. coli lawn and $146.9 \mu \mathrm{m} / \mathrm{s}(+14 \%)$ on plates with no lawn; Table 2). However, it is important to note that in the study of de Bono and Bargmann (1998), each average value represented the average speed of locomotion of at least 24 animals investigated on an unknown number of plates during more than $72 \mathrm{~min}$ of recording, and in the study of de Bono et al. (2002) each average value represented the average speed of locomotion of at least 25 animals investigated on an unknown number of plates during $4 \mathrm{~min}$ of recording. In the present study, the average values of the $\mathrm{N} 2$ worms represented the average speed of locomotion during $60 \mathrm{~s}$ of recording of 172 worms investigated on 10 plates with an E. coli lawn and 173 worms investigated on 10 plates with no lawn. Unfortunately, de Bono and Bargmann (1998) and de Bono et al. (2002) did not provide essential information about the video recording settings used in their experiments (i.e., the magnification of the objective lens, resolution of the digital camera, scale of the pixels, and size of the field-of-view of the camera). Thus, one cannot determine the relation between the size of the field-of-view of the camera and the size of the circular area in which worms could move during the recording time [this area had a diameter of $2.5 \mathrm{~cm}$ in the study of de Bono and Bargmann (1998) and $2.0 \mathrm{~cm}$ in the study of de Bono et al. (2002)]. As a result, it remains unknown how worms were handled that moved out of the field-of-view (or moved into the field-of-view, respectively) of the camera particularly during the long recording time of $72 \mathrm{~min}$ in the study of de Bono and Bargmann (1998). Because de Bono and Bargmann (1998) and de Bono et al. (2002) did not report standard deviations of speed of movement or potential alterations in speed of movement over 
TABLE 4 | Summary of correlation between analyses for worms on plates with no lawn.

\begin{tabular}{|c|c|c|c|c|c|c|c|c|c|}
\hline \multirow[t]{2}{*}{ Calculation } & \multirow[t]{2}{*}{ Strain } & \multicolumn{3}{|c|}{ Runs test } & \multicolumn{3}{|c|}{ Linear regression } & \multicolumn{2}{|c|}{ Non-linear correlation } \\
\hline & & Points above line & Points below line & $p$ & $r^{2}$ & $\boldsymbol{F}$ & $p$ & Spearman & $p$ \\
\hline \multirow{4}{*}{$\begin{array}{l}\text { Average speed vs. average } \\
\text { angle }\end{array}$} & N2 & 73 & 100 & 0.032 & & & & -0.2428 & 0.001 \\
\hline & npr-1(n1353) & 71 & 88 & 0.680 & 0.316 & 72.63 & $<0.001$ & & \\
\hline & npr-1(ad609) & 59 & 80 & 0.010 & & & & 0.085 & 0.323 \\
\hline & npr-1(ky13) & 69 & 98 & 0.088 & 0.164 & 32.31 & $<0.001$ & & \\
\hline \multirow{4}{*}{$\begin{array}{l}\text { Average speed vs. St. Dev. } \\
\text { angle }\end{array}$} & N2 & 74 & 99 & 0.006 & & & & -0.198 & 0.009 \\
\hline & $n p r-1(n 1353)$ & 70 & 89 & 0.172 & 0.059 & 9.913 & 0.002 & & \\
\hline & npr-1(ad609) & 70 & 69 & $<0.001$ & & & & 0.207 & 0.015 \\
\hline & npr-1(ky13) & 67 & 100 & 0.177 & 0.076 & 13.62 & $<0.001$ & & \\
\hline \multirow{4}{*}{$\begin{array}{l}\text { Average speed vs. average } \\
\text { wavelength }\end{array}$} & N2 & 88 & 85 & 0.501 & 0.044 & 7.806 & 0.006 & & \\
\hline & npr-1(n1353) & 83 & 76 & 0.873 & 0.193 & 37.48 & $<0.001$ & & \\
\hline & npr-1(ad609) & 67 & 72 & 0.639 & 0.065 & 9.456 & 0.003 & & \\
\hline & npr-1(ky13) & 77 & 90 & 0.593 & 0.170 & 33.80 & $<0.001$ & & \\
\hline \multirow{4}{*}{$\begin{array}{l}\text { Average speed vs. St. Dev. } \\
\text { wavelength }\end{array}$} & N2 & 82 & 91 & 0.233 & 0.220 & 48.34 & $<0.001$ & & \\
\hline & $n p r-1(n 1353)$ & 82 & 77 & 0.901 & 0.178 & 33.94 & $<0.001$ & & \\
\hline & npr-1(ad609) & 67 & 72 & 0.887 & 0.067 & 9.837 & 0.002 & & \\
\hline & npr-1(ky13) & 79 & 88 & 0.900 & 0.163 & 32.20 & $<0.001$ & & \\
\hline \multirow{4}{*}{$\begin{array}{l}\text { Average speed vs. omega } \\
\text { bending Pct }\end{array}$} & N2 & 44 & 129 & 0.037 & & & & -0.130 & 0.089 \\
\hline & $n p r-1(n 1353)$ & 54 & 105 & 0.198 & 0.060 & 9.997 & 0.002 & & \\
\hline & npr-1(ad609) & 45 & 94 & 0.054 & $<0.0001$ & 0.070 & 0.791 & & \\
\hline & npr-1(ky13) & 30 & 137 & 0.153 & 0.043 & 7.466 & 0.007 & & \\
\hline \multirow{4}{*}{$\begin{array}{l}\text { Average speed vs. reversal } \\
\text { Pct }\end{array}$} & N2 & 63 & 110 & 0.029 & & & & -0.390 & $<0.001$ \\
\hline & $n p r-1(n 1353)$ & 69 & 90 & $<0.001$ & & & & -0.624 & $<0.001$ \\
\hline & npr-1(ad609) & 68 & 71 & 0.307 & 0.252 & 46.14 & $<0.001$ & & \\
\hline & npr-1(ky13) & 65 & 102 & $<0.001$ & & & & -0.436 & $<0.001$ \\
\hline
\end{tabular}

P-values smaller than 0.05 are given boldface.

time, the question remains open as to whether worms showed a relatively constant speed of movement during the recording times in these studies or different speed of movement at the beginning, middle, and end of the recording times. In this regard, a study by Swierczek et al. (2011) should be kept in mind in which N2 C. elegans showed a drop in average speed of locomotion from $\sim 230 \mu \mathrm{m} / \mathrm{s}$ immediately after placing worms on agar plates to $\sim 80 \mu \mathrm{m} / \mathrm{s} 10 \mathrm{~min}$ later (mean of eight plates). In the present study, videos were taken immediately after worms were placed on agar plates; however, the average speed of locomotion of N2 C. elegans on plates with an E. coli lawn was only $~ 55 \%$ of the results reported by Swierczek et al. (2011). In summary, in order to ensure reproducibilty of a study analyzing the behavior of C. elegans it is essential to provide information about the video recording settings used in the experiments in sufficient detail to enable comparisons of the outcome of different studies.

\section{Advanced Behavioral Analysis}

In order to offer advanced C. elegans behavioral analysis, full worm "skeleton and outline" tracing must be present in order to represent full worm behavior and posture rather than only the simple centroid. Some worm trackers described in the literature offer these capabilities, however most trackers that offer skeleton and outline tracking can track only one worm at a time, while most trackers that can track multiple worms offer only centroid tracking (Table 1). With these capabilities, the advanced behavioral analyses performed in the present study are either, in the latter case, not possible, or in the former case must be done by tracking only one worm at a time. As demonstrated here, large interindividual variability creates the need for a large sample size to reliably compare test groups, making a low-throughput, one-worm-at-a-time solution ineffective due to the time required to generate an adequate sample size. In two systems, Multi 
Worm Tracker (Swierczek et al., 2011) and WormLab (Roussel et al., 2014), both multi-worm tracking and skeleton and outline tracking are provided together.

In the present study, the video recording time was restricted to $60 \mathrm{~s}$ in order to limit worms moving out of or moving back into the field-of-view of the camera. However, it was not possible to decrease the magnification at which the videos were recorded. The latter would have resulted in an increased scale of the pixels and, thus, a decreased number of pixels per worm, preventing precise and accurate advanced behavioral analysis as outlined in the present study. In this regard it should be noted that Swierczek et al. (2011) used a camera with a resolution of 2352 $\times 1728$ pixels and performed video tracking of C. elegans at a scale of $24.3 \mu \mathrm{m} /$ pixel. Accordingly, the field-of-view was 24 $\mathrm{cm}^{2}$ in their study and, thus, larger than the base area of the $5 \mathrm{~cm}$ NGM-agar plates used by Swierczek et al. (2011). This is substantially different from the settings used in the present study. Swierczek et al. (2011) reported a position jitter of only $1 \mu \mathrm{m}$ and a speed jitter of only $1 \mu \mathrm{m} / \mathrm{s}$ when analyzing moving C. elegans using these settings, which is similar to what was reported for the WormLab software (Roussel et al., 2014). On the other hand, Swierczek et al. (2011) reported that identity of worms was lost upon collision with another worm, which is mitigated using the settings used in the present study (c.f. Supplemental Video 2 in Angstman et al., 2015).

\section{Measurement of Subtle Behavioral Change}

By measuring behavioral change of worms on plates with and without the presence of $E$. coli, it was demonstrated that certain C. elegans strains demonstrate substantially increased grouping behavior in the presence of $E$. coli (de Bono et al., 2002). Furthermore, the same study showed reduction in speed in multiple C. elegans strains when exposed to food, while also showing that certain deletion mutations affected this phenomenon. In this case, behavioral change was able to shed light on the roles of individual proteins on C. elegans behavioral phenomena.

Further analysis of behavioral change of N2 worms in the presence of an E. coli lawn vs. no lawn has been demonstrated using tracking of individual worms (Schwarz et al., 2015). It should be noted that this methodology differs from that of the present study in a number of ways: (i) individual worms are used rather than groups of worms on plates, (ii) worms on plates are allowed to habituate for a $30 \mathrm{~min}$ period before assaying, while no habituation time is allowed in the present study (in both studies, worms on plates with no lawn are assayed immediately), and (iii) video recording is performed for 15 min, while in the present study video recording consisted of $1 \mathrm{~min}$. In-depth measurement of individual C. elegans posture and its change over time, as demonstrated in Schwarz et al. (2015), could be useful, for example, in the understanding of the neurological functions causing various behaviors, but differences in the methodology used between this and the present study demonstrate two differing scopes.

The present study shows that behavioral change, using the example of $E$. coli lawn presence and four different $C$. elegans strains, can go far beyond simple speed of locomotion measurement to further parameters requiring more in-depth analysis (Table 2). The fact that some of the measured behavioral traits were either only weakly or not at all tied to speed of locomotion (Tables 3, 4) demonstrates the potential utility of expanded data analyses in advanced analysis of C. elegans behavior. In turn, increased behavioral analyses allow for the quantification of behavioral change that may not sufficiently be represented by speed of locomotion. This can be used in combination with knockout $C$. elegans to increase sensitivity in detecting subtle phenotypic changes resulting from genotypic changes. In turn, this could then help in furthering the connection in C. elegans between genetics and behavior.

\section{Nested Analysis}

Due to the design of the experiment requiring the assaying of multiple worms per NGM-agar plate, nested analysis was used to investigate the potential impact of inter-plate variability on statistical analysis, an issue recently reviewed in the literature (Aarts et al., 2014). As summarized in Table 2, the nested factor played a statistically significant role in the observed difference between variables in nearly half $(13 / 28)$ of the analyses. It has previously been suggested that, due to the high interindividual variability observed in C. elegans behavioral assays, a large sample number is required (Angstman et al., 2015). The present study shows that, when using a nested design to accomplish such a sample number, such a design must be accounted for due to inter-plate variability (see also Figure 2). Turned around, if this factor is now accounted for, an even greater number of C. elegans worms must be assayed to achieve the proper statistical power.

\section{Relevance in Toxicology, Drug Discovery, and RNAi Screening}

The methods used in the present study also offer particular relevance in the fields of toxicology, drug discovery, and RNAi screening. Because the effects of various chemical compounds may not be predictable, the ability to screen using multiple parameters offers increased sensitivity in terms of identifying subtle behavioral change. Furthermore, potential changes in behavioral traits in C. elegans may be better defined and measured on agar plates rather than on worms swimming in liquid. Although novel worm tracking software for tracking C. elegans swimming in liquid have recently been published (Hardaway et al., 2014; Restif et al., 2014), most assays involving chemical exposure and the measurement of behavior were performed via chemicals mixed in with the agar. This is, however, not the ideal delivery method for C. elegans as it may require substantially increased concentrations to achieve an effect. This is due to the fact that C. elegans have a cuticle barrier that can result in internal concentrations on the order of magnitudes lower than external concentrations (Rand and Johnson, 1995; Davies et al., 2003; Davies and McIntire, 2004). Exposure to chemicals in liquid, however, provides a much more effective delivery method. This may also apply to RNAi screening, as the ability to carry out RNAi in C. elegans via soaking is less labor intensive than injection and less variable than via agar plate feeding (for details see, e.g., Ahringer, 2006). 
In the present study, worms were maintained solely in a liquid culture until immediately before video capture. Importantly, we have previously demonstrated that C. elegans grown in liquid cultures do not demonstrate statistically significant difference in average speed of locomotion compared to those raised on NGM-agar plates (Angstman et al., 2015). Due to our rapid transfer method, previously described in Angstman et al. (2015), both advantageous scenarios can be achieved: chemical exposure in liquid and behavioral analysis on NGM-agar plates. The combination of this and enhanced capability of behavioral analysis provides an ideal model for use in the fields of toxicology, drug discovery, and RNAi screening.

In summary, the advanced analysis model of C. elegans behavior presented in the present study demonstrates the potential utility and effectiveness of advanced behavioral analyses. Such analyses go beyond simple speed of locomotion measurements, offering greater sensitivity in measuring $C$. elegans behavioral change. This model potentially offers utility in the connecting of C. elegans genetics with behavior as well as in the fields of toxicology, drug discovery, and RNAi screening, to mention only a few.

\section{REFERENCES}

Aarts, E., Verhage, M., Veenvliet, J. V., Dolan, C. V., and van der Sluis, S. (2014). A solution to dependency: using multilevel analysis to accommodate nested data. Nat. Neurosci. 17, 491-496. doi: 10.1038/nn.3648

Ahringer, J. (2006). "Reverse genetics," in WormBook, ed V. Ambros (Cambridge: The C. elegans Research Community). doi: 10.1895/wormbook.1.47.1

Albrecht, D. R., and Bargmann, C. I. (2011). High-content behavioral analysis of Caenorhabditis elegans in precise spatiotemporal chemical environments. Nat. Meth. 8, 599-605. doi: 10.1038/ nmeth. 1630

Angstman, N. B., Kiessling, M. C., Frank, H. G., and Schmitz, C. (2015). High interindividual variability in dose-dependent reduction in speed of movement after exposing C. elegans to shock waves. Front. Behav. Neurosci. 9:12. doi: 10.3389/fnbeh.2015.00012

Buckingham, S. D., and Sattelle, D. B. (2008). Strategies for automated analysis of C. elegans locomotion. Invert. Neurosci. 8, 121-131. doi: 10.1007/s10158-0080077-3

Cleveland, W. S., and Devlin, S. J. (1988). Locally weighted regression: an approach to regression analysis by local fitting. J. Am. Stat. Assoc. 83, 596-610. doi: 10.1080/01621459.1988.10478639

Davies, A. G., and McIntire, S. L. (2004). Using C. elegans to screen for targets of ethanol and behavior-altering drugs. Biol. Proced. 6, 113-119. doi: 10.1251/bpo79

Davies, A. G., Pierce-Shimomura, J. T., Kim, H., VanHoven, M. K., Thiele, T. R., Bonci, A., et al. (2003). A central role of the BK potassium channel in behavioral responses to ethanol in C. elegans. Cell 115, 655-666. doi: 10.1016/S00928674(03)00979-6

de Bono, M., and Bargmann, C. I. (1998). Natural variation in a neuropeptide Y receptor homolog modifies social behavior and food response in C. elegans. Cell 94, 679-689. doi: 10.1016/S0092-8674(00)81609-8

de Bono, M., Tobin, D. M., Davis, M. W., Avery, L., and Bargmann, C. I. (2002). Social feeding in Caenorhabditis elegans is induced by neurons that detect aversive stimuli. Nature 419, 899-903. doi: 10.1038/nature01169

Faumont, S., Rondeau, G., Thiele, T. R., Lawton, K. J., McCormick, K. E., Sottile, M., et al. (2011). An image-free opto-mechanical system for creating virtual environments and imaging neuronal activity in freely moving Caenorhabditis elegans. PLoS ONE 6:e24666. doi: 10.1371/journal.pone.0024666

\section{AUTHOR CONTRIBUTIONS}

NA, HF, and CS conceived and designed the experiments, NA performed experiments, and NA, HF, and CS analyzed data and wrote the manuscript.

\section{ACKNOWLEDGMENTS}

The authors wish to thank Pia Unterberger (Dept. Neuroanat., Ludwig-Maximilians University of Munich) for expert technical assistance.

\section{SUPPLEMENTARY MATERIAL}

The Supplementary Material for this article can be found online at: http://journal.frontiersin.org/article/10.3389/fnbeh. 2016.00060

Video S1 | Wild type (N2) C. elegans on an NGM-agar plate with an E. coli lawn.

Video S2 | Wild type (N2) C. elegans on an NGM-agar plate with no lawn.

Table S1 | Tracking settings used in WormLab software.

Geng, W., Cosman, P., Berry, C. C., Feng, Z., and Schafer, W. R. (2004). Automated tracking, feature extraction and classification of C. elegans phenotypes. IEEE Trans. Biomed. Eng. 15, 1811-1820. doi: 10.1109/TBME.2004.831532

Hardaway, J. A., Wang, J., Fleming, P. A., Fleming, K. A., Whitaker, S. M., Nackenoff, A., et al. (2014). An open-source analytical platform for analysis of C. elegans swimming-induced paralysis. J. Neurosci. Meth. 232, 58-62. doi: 10.1016/j.jneumeth.2014.04.024

Huang, K. M., Cosman, P., and Schafer, W. R. (2006). Machine vision based detection of omega bends and reversals in C. elegans. J. Neurosci. Meth. 158, 323-336. doi: 10.1016/j.jneumeth.2006.06.007

Husson, S. J., Costa, W. S., Schmitt, C., and Gottschalk, A. (2012). "Keeping track of worm trackers," in WormBook, ed O. Hobert (Frankfurt: The C. elegans Research Community). doi: 10.1895/wormbook.1.156.1

Jorgensen, E. M., and Mango, S. E. (2002). The art and design of genetic screens: Caenorhabditis elegans. Nat. Rev. Genet. 3, 356-369. doi: 10.1038/nrg794

Leifer, A. M., Fang-Yen, C., Gershow, M., Alkema, M. J., and Samuel, A. D. (2011). Optogenetic manipulation of neural activity in freely moving Caenorhabditis elegans. Nat. Meth. 8, 147-152. doi: 10.1038/nmeth.1554

Ramot, D., Johnson, B. E., Berry, T. L. Jr., Carnell, L., and Goodman, M. B. (2008). The parallel worm tracker: a platform for measuring average speed and drug-induced paralysis in nematodes. PLOS ONE 3:e2208. doi: 10.1371/journal.pone.0002208

Rand, J. B., and Johnson, C. D. (1995). Genetic pharmacology: interactions between drugs and gene products in Caenorhabditis elegans. Meth. Cell Biol. 48, 187-204. doi: 10.1016/ S0091-679X(08)61388-6

Restif, C., Ibáñez-Ventoso, C., Vora, M. M., Guo, S., Metaxas, D., and Driscoll, M. (2014). CeleST: computer vision software for quantitative analysis of $C$. elegans swim behavior reveals novel features of locomotion. PLoS Comput. Biol. 10:e1003702. doi: 10.1371/journal.pcbi.1003702

Roussel, N., Sprenger, J., Tappan, S. J., and Glaser, J. R. (2014). Robust tracking and quantification of C. elegans body shape and locomotion through coiling, entanglement, and omega bends. Worm 3:e982437. doi: 10.4161/21624054.2014.982437

Schwarz, R. F., Branicky, R., Grundy, L. J., Schafer, W. R., and Brown, A. E. X. (2015). Changes in postural syntax characterize sensory modulation and natural variation of C. elegans locomotion. PLoS Comput. Biol. 11:e1004322. doi: 10.1371/journal.pcbi.1004322 
Stirman, J. N., Crane, M. M., Husson, S. J., Wabnig, S., Schultheis, C., Gottschalk, A., et al. (2011). Real-time multimodal optical control of neurons and muscles in freely behaving Caenorhabditis elegans. Nat. Meth. 8, 153-158. doi: 10.1038/nmeth.1555

Sulston, J. E., and Brenner, S. (1974). The DNA of Caenorhabditis elegans. Genetics 77, 95-104.

Swierczek, N. A., Giles, A. C., Rankin, C. H., and Kerr, R. A. (2011). Highthroughput behavioral analysis in C. elegans. Nat. Meth. 8, 592-598. doi: 10.1038/nmeth.1625

Tsibidis, G. D., and Tavernarakis, N. (2007). Nemo: a computational tool for analyzing nematode locomotion. BMC Neurosci. 8:86. doi: 10.1186/1471-22028-86

Wald, A., and Wolfowitz, J. (1940). On a test whether two samples are from the same population. Ann. Math. Statist. 11, 147-162. doi: 10.1214/aoms/1177731909

White, J. G., Southgate, E., Thomson, J. N., and Brenner, S. (1986). The structure of the nervous system of the nematode Caenorhabditis elegans. Philos. Trans. $R$. Soc. Lond. B. Biol. Sci. 314, 1-340. doi: 10.1098/rstb.1986.0056
Yemini, E., Jucikas, T., Grundy, L. J., Brown, A. E., and Schafer, W. R. (2013). A database of Caenorhabditis elegans behavioral phenotypes. Nat. Methods 10, 877-879. doi: 10.1038/nmeth. 2560

Conflict of Interest Statement: CS serves as paid consultant for MBF Bioscience (Williston, VT, USA), the manufacturer of the WormLab software that was used in the present study to analyze data. However, CS has not received financial support directly or indirectly related to this manuscript. The authors declare that the research was conducted in the absence of any commercial or financial relationships that could be construed as a potential conflict of interest.

Copyright (C) 2016 Angstman, Frank and Schmitz. This is an open-access article distributed under the terms of the Creative Commons Attribution License (CC BY). The use, distribution or reproduction in other forums is permitted, provided the original author(s) or licensor are credited and that the original publication in this journal is cited, in accordance with accepted academic practice. No use, distribution or reproduction is permitted which does not comply with these terms. 